\title{
Medicine and artificial intelligence: a strategy for the future, employing Porter's classic framework
}

Singapore Med J 2020; 61(8): 447 https://doi.org/10.11622/smedj.2019047

Dear Sir,

Medical doctors are not newcomers to the field of artificial intelligence (AI), with radiologists pioneering work in medical imaging perception in the 1980s. ${ }^{(1)}$ But as it stands today, radiology, and in fact the whole of healthcare, is a field ripe for disruption. In the past 5-10 years, there have been substantial new innovations in imaging from deep learning methods of image detection and classification. Current artificial neural networks have accuracy rates that surpass those of radiologists in narrow-based tasks such as nodule detection, ${ }^{(2,3)}$ and those of pathologists in detecting lymph node metastasis from breast cancer, ${ }^{(4)}$ and that are likely close to parity with ophthalmologists for vision-threatening diabetic retinopathy, with an area under the curve of 0.958 (95\% confidence interval 0.956-0.961). ${ }^{(5)}$ Natural language processing is also poised to transform the way electronic health records are utilised by clinicians and researchers.

In the late 1970s, Michael Porter described his classic framework and generic strategies for competitive advantage, which would go on to revolutionise the field of industrial strategy. ${ }^{(6)}$ The three generic strategies determining competitive advantage are cost reduction, differentiation and focus. How do we address these challenges using Porter's strategies? Rather than focusing on a business, let us reframe the point of reference around healthcare as the organisation that requires a strategic rethink. Key to formulating a strategy is defining our capabilities and identifying competitive forces that pose a threat. We face competition from private healthcare contractors who may purchase $\mathrm{Al}$ technologies to market directly to patients, bypassing doctors entirely. We also face competition from equipment vendors who manufacture diagnostic devices such as computed tomography scanners or digital retinal cameras, aiming to integrate their business vertically in much the way Apple produces its phones by owning each part of the supply chain.

Our greatest strength lies, ironically, not in our ability to accurately detect and classify disease, but in our ability to tap on collective and individual experience to make clinical judgements based on data, and this is where we outshine diagnostic algorithms. This experience curve is not limited to knowledge gained from clinical practice but stems from many years of undergraduate basic medical science training beforehand. Currently, we differentiate ourselves by integrating multimodal streams of data from electronic health records and discussions with other colleagues from different specialties. This forms a very strong entry barrier.

That is not to say that Al technology should be resisted, such as by withholding domain expertise from software developers. On the contrary, our strategic goal should be to further differentiate ourselves by creating hybrid clinicians and Al as a form of collective intelligence. Indeed, it would be unethical, if not impossible, to stunt the progress of clinical medicine by blocking fundamental technology. It should also be noted that one of the modifiers of Porter's competitive forces in industrial economics include government policy, which is a very major consideration when studying the risks posed by new entrants to the market, but this is not addressed here for the sake of brevity.

Apart from thinking of ourselves as a product, we can also position ourselves as buyers to exert a strategic force upon the market and by backward integration: software is much easier to create than machines, and deep learning code is already freely available on an open source basis. Instead of buying expensive Al software, we have been creating our own, in-house.

In the future, a probable strategy is the diversification of medicine into a broader field that utilises all forms of data, including genomics, metadata, electronic health records and biometrics, to arrive at a timely diagnosis around the clock. Building an ecosystem to sustain this, together with other 'information specialists' such as pathologists, ${ }^{(7)}$ would create an even greater competitive advantage. Finally, using the time freed by increased productivity, 'brand' identification may be cultivated in our patients by increasing face-to-face interactions, doing things 'for' rather than 'to' our patients and fostering awareness of our Al-enhanced capabilities that make medicine safer, more precise and more patient-focused, with the aim of establishing clinicians augmented with Al as the future gold standard.

Yours sincerely,

Charlene JY Liew

Department of Radiology, Changi General Hospital. charlene_liew@cgh.com.sg

\section{References}

1. Krupinski EA. The future of image perception in radiology: synergy between humans and computers. Acad Radiol 2003; 10:1-3.

2. Wang H, Zhao T, Li LC, et al. A hybrid CNN feature model for pulmonary nodule malignancy risk differentiation. J Xray Sci Technol 2018; 26:171-187.

3. Becker AS, Marcon M, Ghafoor S, et al. Deep learning in mammography: diagnostic accuracy of a multipurpose image analysis software in the detection of breast cancer. Invest Radiol 2017; 52:434-40.

4. Ehteshami Bejnordi B, Veta M, Johannes van Diest P, et al. Diagnostic assessment of deep learning algorithms for detection of lymph node metastases in women with breast cancer. JAMA 2017; 318:2199-210.

5. Ting DSW, Cheung CY, Lim G, et al. Development and validation of a deep learning system for diabetic retinopathy and related eye diseases using retinal images from multiethnic populations with diabetes. JAMA 2017; 318:2211-23.

6. Porter ME. How competitive forces shape strategy. Harv Bus Rev 1979; 102-17.

7. Jha S, Topol EJ. Adapting to artificial intelligence: radiologists and pathologists as information specialists. JAMA 2016; 316:2353-4. 